\title{
Blind Channel Estimation in Multi-Rate CDMA Systems
}

\author{
Sumit Roy, Senior Member, IEEE, and Hongbo Yan
}

\begin{abstract}
Multi-rate CDMA is a potentially attractive multiple access method for future broad-band multimedia wireless networks that must support integrated voice/data traffic. The primary impairment for such multi-rate systems is the multipath nature of radio channels that results in intra-user inter-chip interference (ICI) and multi-user interference (MUI) between different users' symbols. Explicit knowledge of the channel is typically needed for high performance detectors (such as coherent demodulation). In this work, we propose a subspace method for channel estimation in multi-rate CDMA systems. A unified signal model that applies to three multi-rate CDMA schemes proposed in the literature is developed. The computational complexity for multi-rate scenarios is large and variable-accordingly, a modified approach is devised that offers performance/complexity trade-offs. Performance analysis is conducted based on a close-form expression for the mean square error of the estimator, supported by simulation results that investigate the effectiveness of our method.
\end{abstract}

Index Terms-Blind channel estimation, code division multiple access, multi-code, multi-rate, variable chip rate, variable processing gain.

\section{INTRODUCTION}

$\mathbf{N}$ EXT generation networks are expected to support multimedia traffic such as voice, video and data. Such heterogeneous information sources are inherently multi-rate in nature. Among the principal candidate multiple access schemes, CDMA offers a relatively straightforward solution to this new communication scenario. It is well matched to bursty multi-rate, multimedia traffic as it allows for dynamic allocation of bandwidth resources among the active users; thus CDMA based air interfaces have been adopted for 3G systems [1], [2]. In the literature, three access methodologies are proposed for multi-rate CDMA - variable chip rate, variable processing gain and multi-code transmission [3]-[5], as shown in Fig. 1. In variable chip rate systems, data streams at different rates are spread with codes of the same length, i.e., different rate users use different chip rates, implying that the available bandwidth is not fully used by the low rate users. Variable processing gain systems avoid this problem by spreading data at different rates over the same bandwidth with codes of different length, resulting in identical chip rate for all the users. The price paid is the loss of processing gain by high rate users. In multi-code systems, all rates are assumed to be multiples of a basic rate.

Paper approved by L. Wei, the Editor for Coding Theory and Applications of the IEEE Communications Society. Manuscript received September 8, 2000; revised May 3, 2001, and November 7, 2001. This work was supported in part by AFOSR under Grant F49620-1-0472.

The authors are with the Department of Electrical Engineering, University of Washington, Seattle, WA 98195-2500 USA (e-mail: roy@ee.washington.edu).

Publisher Item Identifier S 0090-6778(02)05543-5.

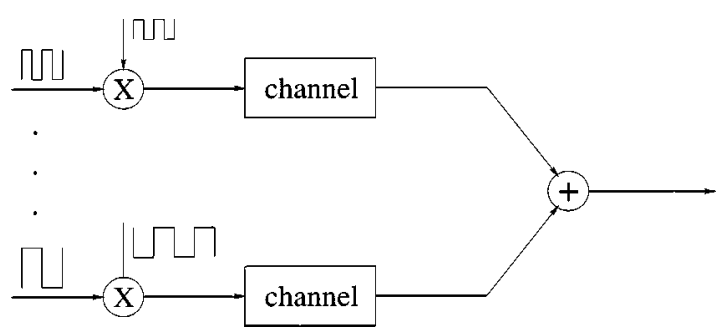

(a)

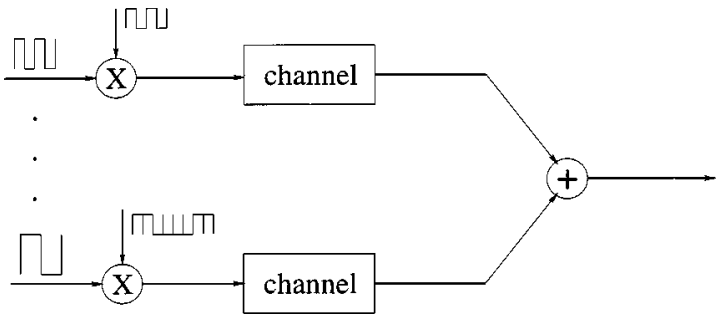

(b)

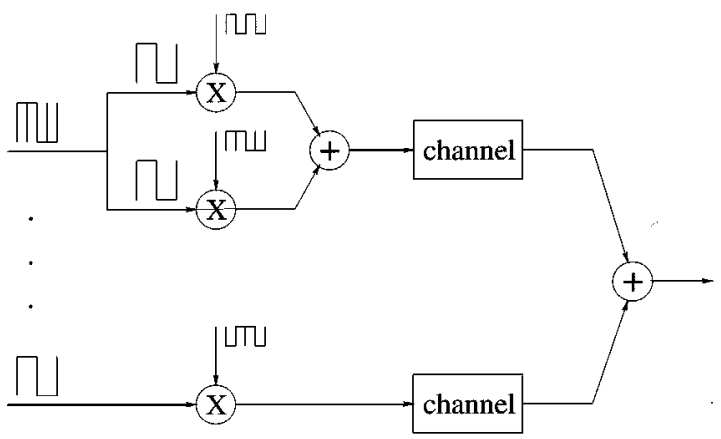

(c)

Fig. 1. Three multi-rate CDMA access methods. (a) Variable chip rate access method. (b) Variable processing gain access method. (c) Multiple code access method.

Each stream is first (serial-to-parallel) down converted into several basic rate streams; these lower rate substreams are then spread over the same bandwidth for transmission. Orthogonal codes are proposed to prevent self-interference between the substreams [3], [6]. However, the presence of a dispersive channel as is common in wireless access results in loss of this orthogonality [15]. To deal with mutual interference between signal components at different rates, one usually needs to collect data samples over multiple symbol duration, implying increased processing time and memory in contrast to the single rate scenario. This provides the main challenge for receiver design in multi-rate CDMA systems.

Decorrelating receivers have been devised and analyzed in [7]-[9] for synchronous and asynchronous systems respectively. Successive interference cancellation methods are developed in 


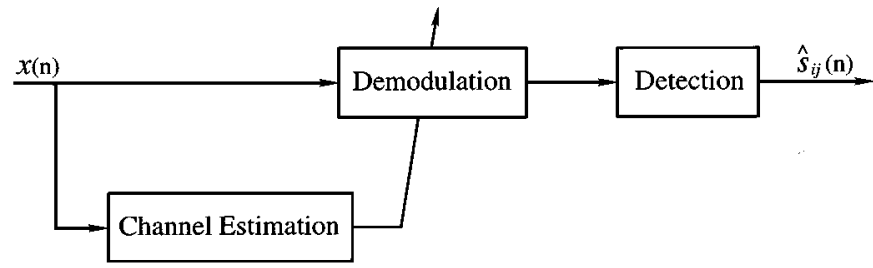

Fig. 2. Multi-rate CDMA detector.

[10]-[12]. While published results are critically dependent on specific codes used in the design, a general consensus is that for the conventional matched filter receiver, variable processing gain and multi-code systems have identical performance [5], [6] while for maximum likelihood detection, variable processing gain is preferable to a comparable multi-code realization [13].

A random code analysis for the dual-rate case is presented in [14] using the optimum near-far resistance (NFR) as the performance measure to compare detector structures. It is shown that for high rate users, optimum NFR for variable processing gain systems is identical to that of variable chip rate and better than multi-code systems; for low rate users, the multi-code systems have same NFR as variable processing gain systems while surpassing variable chip rate systems.

In this paper, we consider multi-rate CDMA signaling through dispersive channels. A general signal reception model is proposed that incorporates all three access methods. The CDMA receiver architecture depicted in Fig. 2 requires channel estimation; in this work, we develop a subspace method for this purpose. This problem has been addressed in [17], [18] for single rate systems; our algorithm is a generalization to the multi-rate case and accommodates single-rate as a special case. Based on a unified data model, we accomplish channel estimation for all three access methods under the same framework. A reduced complexity method is also proposed to lessen computational burden.

The rest of the paper is organized as follows, Section II presents the received signal model; Section III outlines the algorithm for channel estimation and Section IV refines the method for complexity reduction. Simulation results are given in Sections V and VI concludes the work.

Throughout the paper, uppercase letters in boldface or calligraphy denote matrices; lowercase letters in boldface stand for vectors; $(\cdot)^{T},(\cdot)^{H}$ represent transpose and Hermitian respectively; $\|\cdot\|,\|\cdot\|_{F}$ are two-norm and Frobenius norm; $(\cdot)^{\dagger}$ is the pseudo-inverse and $\mathbf{I}_{d}$ is the $d \times d$ unity matrix.

\section{Signal Models AND PRoblem Formulation}

\section{A. Variable Processing Gain Systems}

A baseband variable processing gain multi-rate CDMA communication system can be modeled as

$$
x(t)=\sum_{i=1}^{N} \sum_{j=1}^{K_{i}} r_{i j}(t)+w(t)
$$

where $N$ and $K_{i}$ respectively denote the total number of data rates and the total number of users at rate $i$ and $w(t)$ is additive

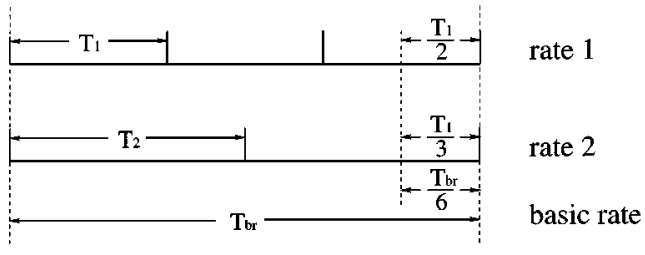

Fig. 3. A two-user variable processing gain system: rate ratio $2: 3$.

white Gaussian noise of variance $\sigma_{w}^{2}$. The signal component $r_{i j}(t)$ due to user $j$ at rate $i$ is given by

$$
r_{i j}(t)=\sum_{k=-\infty}^{\infty} s_{i j}(k) g_{i j}\left(t-k T_{i}\right)
$$

where the zero mean information sequences $s_{i j}(k)$ s are i.i.d., of respective variance $\sigma_{i j}^{2}$ and independent of noise $w(t) ; T_{i}$ is the symbol duration of rate $i$ sequences. The signature waveform $g_{i j}(t)$ as seen by the receiver is the convolution of the channel impulse response $h_{i j}(t)$ and the pre-assigned transmitted spreading code $c_{i j}(l)$ :

$$
g_{i j}(t)=\sum_{l=0}^{L_{i}-1} c_{i j}(l) h_{i j}\left(t-l T_{c}\right)
$$

where $L_{i}$ is the processing gain of rate $i$ users, $T_{c}$ is the chip duration and $h_{i j}(t)$ is the composite channel which includes the fixed transmit/receive pulse shaping filters (e.g., raised cosine pulse) and the unknown multipath physical channel [19]

$$
f_{i j}(t)=\sum_{k=1}^{D_{i j}} \alpha_{k}(t) \delta\left(t-\tau_{k}\right)
$$

where $D_{i j}$ is the number of distinct paths, $\alpha_{k}(t)$ is the complex gain of the path and $\tau_{k}$ is the propagation delay. We point out that for different users these parameters may be different; in this work, we will assume static multipath, i.e., the channel amplitudes and delays are essentially fixed over the observation duration. For practical purposes, $h_{i j}(t)$ can be modeled as a FIR filter [19], [20], which incurs inter-symbol interference (ISI) as shown in the signal model.

Since all the users share a common bandwidth in the variable processing gain systems, the chip duration must be independent of $i$, hence

$$
T_{c}=\frac{T_{1}}{L_{1}}=\cdots=\frac{T_{N}}{L_{N}}
$$

where $L_{i}$ is the processing gain for rate $i$. This indicates

$$
\frac{T_{1}}{p_{1}}=\frac{T_{2}}{p_{2}}=\cdots=\frac{T_{N}}{p_{N}}=\frac{T_{b r}}{P}
$$

where $p_{1}, p_{2}, \ldots, p_{N}$ are co-prime integers which indicate the rate ratio, $P=\prod_{i=1}^{N} p_{i}$ is the least common multiple of $p_{i}$ s and $1 / T_{b r}$ is called basic rate. Introduce for future use $q_{i}=$ $P / p_{i}$, then rate $i$ is $q_{i}$ times the basic rate. Denote $L$ the greatest common divisor of $L_{1}, \ldots, L_{N}$, it is easy to see that $L_{i}=$ $L p_{i}$. Fig. 3 illustrates the relative symbol duration of a dual-rate system where the rate ratio is $2: 3 . P=6$ in this example; rates 1 and 2 are respectively twice and three times the basic rate. 
Sampling the received signal at chip rate, we obtain the discrete time model

$$
x(n)=\sum_{i=1}^{N} \sum_{j=1}^{K_{i}} r_{i j}(n)+w(n)
$$

where

$$
r_{i j}(n)=\sum_{k=-\infty}^{\infty} s_{i j}(k) g_{i j}\left(n-k L_{i}\right)
$$

and

$$
g_{i j}(n)=\sum_{l=0}^{L_{i}-1} c_{i j}(l) h_{i j}(n-l) .
$$

We assume that all equi-rate users have the same channel order $M_{i}$ measured in chip duration (the generalization to the case of distinct channel orders is straightforward). Since generally $M_{i} \ll L_{i}$, we reasonably assume that the duration of $g_{i j}(n)$ is $2 L_{i}$ with some trailing zeros. In matrix form

$$
\begin{aligned}
\mathbf{g}_{i j} & =\left[g_{i j}(0) \cdots g_{i j}\left(2 L_{i}-1\right)\right]^{T} \\
& =\left[\begin{array}{ccc}
c_{i j}(0) & & \\
\vdots & \ddots & \\
c_{i j}\left(L_{i}-1\right) & & c_{i j}(0) \\
& \ddots & \vdots \\
\vdots & & c_{i j}\left(L_{i}-1\right) \\
& & \vdots \\
0 & \cdots & 0
\end{array}\right]\left[\begin{array}{c}
h_{i j}(0) \\
\vdots \\
h_{i j}\left(M_{i}\right)
\end{array}\right] \\
& =\mathbf{C}_{i j} \mathbf{h}_{i j} .
\end{aligned}
$$

For full (column) rank $\mathbf{C}_{i j}, \mathbf{h}_{i j}$ can be uniquely recovered from $\mathrm{g}_{i j}$; thus estimation of the received signature waveform and channel is equivalent.

For the single rate scenario, second order blind identification schemes [22], [23] are based on exploiting the cyclostationarity of the received (continuous-time) signal via oversampling. These methods collect the channel output samples over a symbol duration to yield a vector wide-sense stationary process for subsequent channel estimation. In the multi-rate case, the received signal is composed of several wide-sense cyclostationary component signals with different symbol periods; thus the observation vector length must be (multiples of) the least common multiple of the constituent symbol duration, given by $T=m T_{b r}$. For the example shown in Fig. 3, if there is only one rate in the system, say rate 1 , a snapshot of duration $T_{1}$ is enough to characterize the received signal. In contrast, in the case of two symbol rates, received signal structure repeats every $T_{b r}$ duration, therefore a snapshot of at least that length is needed to describe the system. The coefficient $m$ is called the (block) smoothing factor. Since $T_{b r}=q_{i} T_{i}$, the effective smoothing factor for rate $i$ users is $m q_{i}$ [18].

Collecting chip rate samples $r_{i j}(n)$ over an interval of $T$ yields

$$
\begin{aligned}
\mathbf{r}_{i j}(n) & =\left[r_{i j}\left(n L_{i}\right) \cdots r_{i j}\left(\left(n+m q_{i}\right) L_{i}-1\right)\right]^{T} \\
& =\left[\begin{array}{llll}
\mathbf{g}_{i j}^{2} & \mathbf{g}_{i j}^{1} & & \\
& \ddots & \ddots & \\
& & \mathbf{g}_{i j}^{2} & \mathbf{g}_{i j}^{1}
\end{array}\right]\left[\begin{array}{c}
s_{i j}(n-1) \\
\vdots \\
s_{i j}\left(n+m q_{i}-1\right)
\end{array}\right] \\
& =\mathbf{G}_{i j} \mathbf{s}_{i j}(n)
\end{aligned}
$$

where

$$
\begin{aligned}
& \mathbf{g}_{i j}^{1}=\left[g_{i j}(0) \cdots g_{i j}\left(L_{i}-1\right)\right]^{T}, \\
& \mathbf{g}_{i j}^{2}=\left[g_{i j}\left(L_{i}\right) \cdots g_{i j}\left(2 L_{i}-1\right)\right]^{T} .
\end{aligned}
$$

It is clear that $\mathbf{g}_{i j}^{1}$ characterizes the contribution of the current symbol to the received signal and $\mathbf{g}_{i j}^{2}$ reflects ISI due to the previous symbol. Correspondingly partition $\mathbf{C}_{i j}$ such that

$$
\mathbf{g}_{i j}^{1}=\mathbf{C}_{i j}^{1} \mathbf{h}_{i j}, \quad \mathbf{g}_{i j}^{2}=\mathbf{C}_{i j}^{2} \mathbf{h}_{i j},
$$

then $m L P \times\left(m q_{i}+1\right)$ matrix $\mathbf{G}_{i j}$ can be expressed as

$$
\begin{aligned}
& \mathbf{G}_{i j}=\underbrace{\left[\begin{array}{cccc}
\mathbf{C}_{i j}^{2} & \mathbf{C}_{i j}^{1} & & \\
& \ddots & \ddots & \\
& & \mathbf{C}_{i j}^{2} & \mathbf{C}_{i j}^{1}
\end{array}\right]}_{m L P \times\left(m q_{i}+1\right)\left(M_{i}+1\right)} \underbrace{\left[\begin{array}{lll}
\mathbf{h}_{i j} & & \\
& \ddots & \\
& & \mathbf{h}_{i j}
\end{array}\right]}_{\left(m q_{i}+1\right)\left(M_{i}+1\right) \times\left(m q_{i}+1\right)} \\
&=\mathcal{C}_{i j} \mathcal{H}_{i j}
\end{aligned}
$$

where $\mathcal{H}_{i j}$ is the block diagonal matrix of $\mathbf{h}_{i j}$. The observation vector for this $T$-duration is

$$
\begin{aligned}
\mathbf{x}_{n} & =[x(n L P) \cdots x(n L P+m L P-1)]^{T} \\
& =\sum_{i=1}^{N} \sum_{j=1}^{K_{i}} \mathbf{r}_{i j}\left(n q_{i}\right)+\mathbf{w}_{n} \\
& =\underbrace{\left[\mathbf{G}_{11} \cdots \mathbf{G}_{N K_{N}}\right]}_{m L P \times \sum_{i=1}^{N} K_{i}\left(m q_{i}+1\right)}\left[\begin{array}{c}
\mathbf{s}_{11}\left(n q_{1}\right) \\
\vdots \\
\mathbf{s}_{N K_{N}}\left(n q_{N}\right)
\end{array}\right]+\mathbf{w}_{n} \\
& =\mathbf{G s}_{n}+\mathbf{w}_{n}
\end{aligned}
$$

where noise vector $\mathbf{w}_{n}=[w(n L P) \cdots w(n L P+m L P-1)]^{T}$. Note that the length of the observation vector is a function of the smoothing factor and processing gains. For example, consider a dual-rate system with rate ratio $2: 3$ and $L=15$; the dimension of the observation $\mathrm{x}_{n}$ will be $90 \mathrm{~m}$, indicating a significant numerical complexity. We construct a data matrix $\mathbf{X}$ by placing $J$ successive $\mathbf{x}_{n}$ 's columnwise

$$
\begin{aligned}
\mathbf{X} & =\left[\begin{array}{lll}
\mathbf{x}_{n} & \cdots & \mathbf{x}_{n+J-1}
\end{array}\right] \\
& =\mathbf{G}\left[\begin{array}{lll}
\mathbf{s}_{n} & \cdots & \mathbf{s}_{n+J-1}
\end{array}\right]+\left[\begin{array}{lll}
\mathbf{w}_{n} & \cdots & \mathbf{w}_{n+J-1}
\end{array}\right] \\
& =\mathbf{G S}+\mathbf{W} .
\end{aligned}
$$

The problem addressed in this paper is the estimation of $h_{i j}(n)$ [and therefore $g_{i j}(n)$ ] from $\mathbf{X}$ without explicit knowledge of $\mathbf{S}$.

\section{B. Single Rate and Multi-Code Systems}

It is easy to see that the single rate system is a special case of the variable processing gain multi-rate systems with $N=1$, $p_{N}=1, L_{N}=L, K_{N}=K$ and $M_{N}=M$, for which the snapshot interval $T=m L T_{c}$.

In multi-code systems, multiple data (sub)streams are sent in parallel, each at the basic rate defined in (6). Clearly, a single rate $i$ user can be viewed as $q_{i}$ virtual users at the basic rate (see Fig. 3). In this manner, multi-code systems are equivalent to single rate systems with more (virtual) users and hence (16) still applies. 


\section{Variable Chip Rate Systems}

For variable chip rate multi-rate CDMA systems, the signature waveform is given by

$$
g_{i j}(t)=\sum_{l=0}^{L-1} c_{i j}(l) h_{i j}\left(t-l T_{c}^{i}\right)
$$

where $L$ is the processing gain and thus the chip duration for rate $i$ users is $T_{c}^{i}=T_{i} / L$. Suppose (6) is still valid, then sampling with an interval $\Delta=T_{c}^{i} / p_{i}=T_{i} / L_{i}$ yields

$$
r_{i j}(n)=\sum_{k=-\infty}^{\infty} s_{i j}(k) g_{i j}\left(n-k L_{i}\right)
$$

and

$$
g_{i j}(n)=\sum_{l=0}^{L-1} c_{i j}(l) h_{i j}\left(n-l p_{i}\right) .
$$

It is easy to verify that (19) is equivalent to

$$
g_{i j}(n)=\sum_{l=0}^{L_{i}-1} \psi_{i j}(l) h_{i j}(n-l)
$$

where length $L_{i}$ effective spreading code $\psi_{i j}(n)$ is defined as

$$
\psi_{i j}(n)=\sum_{l=0}^{L-1} c_{i j}(l) \delta\left(n-l p_{i}\right)
$$

Note that (8) and (18) have exactly the same form, as do (9) and (20). Thus we conclude that (16) accommodates variable chip rate systems as well. Clearly, the assumption on rate ratio specified in (6) is a requirement for (18) and (19) to be valid. Hence, we summarize that the unified model covers all three multi-rate CDMA access methods only if (6) holds; this is almost always true in practice, particularly for variable processing gain and multi-code systems.

\section{SIGNATURE WAVEFORM/CHANNEL ESTIMATION}

A method is derived in this section for signature waveform/channel estimation by exploiting the subspace of matrix $\mathbf{X}$ that contains the relevant channel information due to (16). In order for the noise free observation matrix $\mathbf{X}$ to share the same subspace structure as $\mathbf{G}$, a necessary condition is that $\mathbf{S}$ must have full row rank [21]. For $\sum_{i=1}^{N} K_{i}\left(m q_{i}+1\right) \times J$ matrix $\mathbf{S}$ with elements randomly sampled from a finite alphabet, this condition is satisfied with probability 1 as $J$ is increased beyond $\sum_{i=1}^{N} K_{i}\left(m q_{i}+1\right)$ [24]. Thus given symbol rates, system load and the smoothing factor, we obtain the following requirement on the number of observation vectors for the validity of the algorithm.

Lemma 1: A necessary condition for subspace based channel estimation requires $J$ to satisfy $J>\sum_{i=1}^{N} K_{i}\left(m q_{i}+1\right)$.

\section{A. Algorithm Outline}

We first consider the noise free case. Assuming the above necessary condition is satisfied, applying the singular value decomposition (SVD) [25] to data matrix $\mathbf{X}$, we have

$$
\mathbf{X}=\left[\begin{array}{ll}
\mathbf{U}_{s} & \mathbf{U}_{w}
\end{array}\right]\left[\begin{array}{cc}
\boldsymbol{\Lambda}_{s} & \mathbf{0} \\
\mathbf{0} & \mathbf{0}
\end{array}\right]\left[\begin{array}{ll}
\mathbf{V}_{s} & \mathbf{V}_{w}
\end{array}\right]^{H}
$$

The vectors in $\mathbf{U}_{s}$, associated with the singular values in diagonal matrix $\boldsymbol{\Lambda}_{s}$, span the signal subspace defined by the columns of $\mathbf{G}$, and the vectors in $\mathbf{U}_{w}$, associated with the zero singular values, span the orthogonal complement of $\mathbf{U}_{s}$ (and hence of G). Thus we have

$$
\mathbf{U}_{w}^{H} \mathbf{G}=\mathbf{0}
$$

Since $\mathbf{G}=\left[\cdots \mathbf{G}_{i j} \cdots\right]$, this yields

$$
\mathbf{U}_{w}^{H} \mathbf{G}_{i j}=\mathbf{0}, \quad 1 \leq i \leq N ; 1 \leq j \leq K_{i} .
$$

Denoting $\mathcal{C}_{i j}^{l}\left(1 \leq l \leq m q_{i}+1\right)$ as the submatrix of $\mathcal{C}_{i j}$ in (14) consisting of columns $(l-1)\left(M_{i}+1\right)+1$ through $l\left(M_{i}+1\right)$, then according to (14), (24) can be combined into

$$
\underbrace{\left[\begin{array}{ccc}
\mathbf{U}_{w} & H & \\
& \ddots & \\
& & \mathbf{U}_{w}
\end{array}\right]}_{\tilde{\mathbf{U}}_{w}^{H}} \underbrace{\left[\begin{array}{c}
\mathcal{C}_{i j}^{1} \\
\vdots \\
\mathcal{C}_{i j}^{m q_{i}+1}
\end{array}\right]}_{\tilde{\mathbf{C}}_{i j}} \mathbf{h}_{i j}=\mathbf{Q}_{i j} \mathbf{h}_{i j}=\mathbf{0}
$$

where $\tilde{\mathbf{U}}_{w}$ is a block diagonal matrix with block element $\mathbf{U}_{w}$. Equation (25) yields a set of $\left(m q_{i}+1\right)(m L P-\operatorname{Rank}(\mathbf{G}))$ linear equations with $M_{i}+1$ unknowns. To determine $\mathbf{h}_{i j}$ up to a scalar,

$$
\operatorname{Rank}\left(\mathbf{Q}_{i j}\right)=M_{i}
$$

needs to be satisfied. A necessary condition for this is

$$
\left(m q_{i}+1\right)(m L P-\operatorname{Rank}(\mathbf{G})) \geq M_{i} .
$$

Assuming that $\mathbf{G}$ has full rank, we arrive at

$$
\left(m q_{i}+1\right)\left(m L P-\sum_{i=1}^{N} K_{i}\left(m q_{i}+1\right)\right) \geq M_{i}
$$

Thus together with Lemma 1, we obtain

Theorem 1: For any rate $i$, a set of necessary conditions for channel estimation with the proposed algorithm are

1) $J>\sum_{i=1}^{N} K_{i}\left(m q_{i}+1\right)$;

2) $\left(m q_{i}+1\right)\left(m L P-\sum_{i=1}^{N} K_{i}\left(m q_{i}+1\right)\right) \geq M_{i}$.

It is observed in [18] that better performance is generally expected with larger smoothing factors but with diminishing returns when the smoothing factor exceeds 3 . In our case, the effective smoothing factor $m q_{i}$ is usually no less than 2 . Since the numerical complexity of the proposed algorithm is a quadratic function of $m$, as will been seen in Section IV, we propose to choose the smallest $m$ which satisfies the above conditions.

In the presence of noise, since only a perturbed version of $\mathbf{Q}_{i j}$ can be obtained, (25) is replaced by the LS criterion

$$
\hat{\mathbf{h}}_{i j}=\arg \min _{\left\|\mathbf{h}_{i j}\right\|=1}\left\|\mathbf{Q}_{i j}^{H} \mathbf{h}_{i j}\right\|^{2}
$$

which provides an estimate of $\mathbf{h}_{i j}$ up to an unknown scalar. The signature waveform estimate $\hat{\mathbf{g}}_{i j}$ can then be determined from (10). 


\section{B. Identifiability}

The identifiability condition from (25) is that there exists a $\left(M_{i}+1\right) \times 1$ vector $\mathbf{h}$ such that

$$
\tilde{\mathbf{g}}=\tilde{\mathbf{C}}_{i j} \mathbf{h}
$$

lies both in $\operatorname{Range}\left(\tilde{\mathbf{C}}_{i j}\right)$ and $\operatorname{Null}\left(\tilde{\mathbf{U}}_{w}^{H}\right)$. Let $\tilde{\mathbf{G}}=$ $\operatorname{diag}[\mathbf{G} \cdots \mathbf{G}]$, then clearly $\operatorname{Null}\left(\tilde{\mathbf{U}}_{w}^{H}\right)=\operatorname{Range}(\tilde{\mathbf{G}})$. Since the columns of $\tilde{\mathbf{C}}_{i j}$ are independent, the mapping of (30) is isomorphic. Thus the identifiability condition becomes

$$
\operatorname{Dim}\left(\operatorname{Range}\left(\tilde{\mathbf{C}}_{i j}\right) \bigcap \operatorname{Range}(\tilde{\mathbf{G}})\right)=1
$$

for uniqueness of the solution to (25), which is re-stated as follows.

Theorem 2: $\mathbf{h}_{i j}$ can be uniquely determined up to some scalar with the algorithm iff the intersection between $\operatorname{Range}\left(\tilde{\mathbf{C}}_{i j}\right)$ and $\operatorname{Range}(\tilde{\mathbf{G}})$ is one-dimensional.

An interesting observation from the theorem is that $\mathbf{G}$ does not need to be full rank for the channel to be identifiable, which is in contrast to the conclusions for non-CDMA systems [23]. With partial information of $\tilde{\mathbf{g}}$ - the user spreading code, the channel can be uniquely determined when the identifiability condition is satisfied.

\section{Channel Order Selection}

In the above derivation, we assume that the channel orders $M_{i}$ are known a priori at the receiver. Sensitivity of the method to such knowledge is a key aspect of algorithm performancefrom (25), it follows that overestimation of the channel order will only introduce additional zeros in the tail of the vector $\mathbf{h}_{i j}$. Thus the subspace based channel estimator is expected to be robust against channel order overestimation-in practice, one can use an estimate based on the maximum anticipated delay spread at the cost of some performance degradation in channel estimates.

\section{REDUCED COMPLEXITY METHOD}

The computational burden of the above algorithm is due to a full SVD on $m L P \times J$ data matrix $\mathbf{X}$. Defining $a=\max (m L P, J)$ and $b=\min (m L P, J)$, such an operation requires $O\left(a^{2} b\right)$ flops [25]. Since $L P$ is a function of the rates and is usually much larger than the system's processing gains, multi-rate systems incur costs that may not be acceptable in practice. To gain insight into this complexity, we compare three dual-rate scenarios. The first system has rate ratio of $2: 3$ and $L=15$; for the second, the rate ratio is $7: 11$ and $L=4$ to keep the processing gains roughly the same; the third system has rate ratio $2: 5$ and $L=15$ such that the high rate users' processing gain is the same. Supposing $m L P>J$, the complexities are $O\left((90 m)^{2} J\right), O\left((308 m)^{2} J\right)$ and $O\left((150 m)^{2} J\right)$ in the three cases, respectively. In this section, we explore ways to reduce this complexity by trading off performance; a direct method is to use a reduced dimension observation vector in place of $\mathbf{x}_{n}$. Introduce the new notation $\mathbf{A}\left(l_{1}: l_{2}\right)\left(\mathbf{a}\left(l_{1}: l_{2}\right)\right)$ for the submatrix of $\mathbf{A}$ (subvector of a) consisting of rows (entries) $l_{1}$ through $l_{2}$. It is clear from (16) that

$$
\mathbf{X}\left(l_{1}: l_{2}\right)=\mathbf{G}\left(l_{1}: l_{2}\right) \mathbf{S}+\mathbf{W}\left(l_{1}: l_{2}\right) .
$$

We show the row dimension $d_{r}=l_{2}-l_{1}+1$ of $\mathbf{X}\left(l_{1}: l_{2}\right)$ may be chosen so as to achieve considerable computation savings compared to the full dimension case, when $L P$ is large.

Applying SVD to noise free data matrix $\mathbf{X}\left(l_{1}: l_{2}\right)$ yields

$$
\mathbf{X}\left(l_{1}: l_{2}\right)=\left[\begin{array}{ll}
\mathcal{U}_{s} & \mathcal{U}_{w}
\end{array}\right]\left[\begin{array}{cc}
\boldsymbol{\Gamma}_{s} & \mathbf{0} \\
\mathbf{0} & \mathbf{0}
\end{array}\right]\left[\begin{array}{ll}
\mathcal{V}_{s} & \mathcal{V}_{w}
\end{array}\right]^{H}
$$

It follows, as before that

$$
\mathcal{U}_{w}^{H} \mathbf{G}_{i j}\left(l_{1}: l_{2}\right)=\mathcal{U}_{w}^{H} \mathcal{C}_{i j}\left(l_{1}: l_{2}\right) \mathcal{H}_{i j}=\mathbf{0} .
$$

Denote by $\mathcal{C}_{i j}^{l}\left(l_{1}: l_{2}\right)\left(1 \leq l \leq m q_{i}+1\right)$ the submatrix of $\mathcal{C}_{i j}\left(l_{1}: l_{2}\right)$ consisting of columns $(l-1)\left(M_{i}+1\right)+1$ through $l\left(M_{i}+1\right)$, (34) can be rewritten as

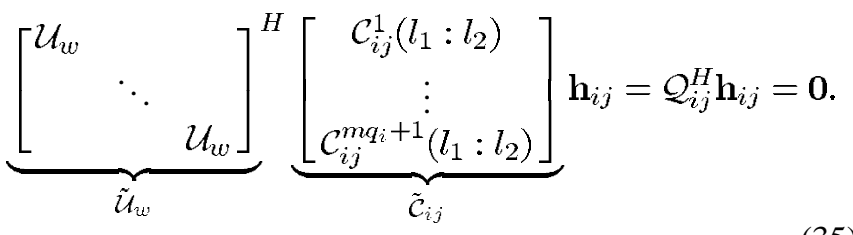

Then in the noisy case $\mathbf{h}_{i j}$ is estimated from

$$
\hat{\mathbf{h}}_{i j}=\arg \min _{\left\|\mathbf{h}_{i j}\right\|=1}\left\|\mathcal{Q}_{i j}^{H} \mathbf{h}_{i j}\right\|^{2}
$$

where it is clear that the modified method includes the original for $l_{1}=1, l_{2}=m L P$.

Note that there may be all-zero columns in $\mathbf{G}\left(l_{1}: l_{2}\right)$ for some $l_{1}, l_{2}$ pairs. Letting $d_{r}=l_{2}-l_{1}+1$, the number of rows in $\mathbf{G}\left(l_{1}: l_{2}\right)$ and $d_{c}$ the number of nonzero columns in $\mathbf{G}\left(l_{1}: l_{2}\right)$, we obtain the following:

Theorem 3: For any rate $i$, the necessary conditions for the refined method are

1) $J>d_{c}$;

2) $\left(m q_{i}+1\right)\left(d_{r}-d_{c}\right) \geq M_{i}$.

The identifiability condition is stated below.

Theorem 4: $\mathbf{h}_{i j}$ can be uniquely determined up to some scalar with the reduced complexity method iff the intersection between Range $\left(\tilde{\mathcal{C}}_{i j}\right)$ and Range $(\tilde{\mathcal{G}})$ is one-dimensional, where $\tilde{\mathcal{G}}=\operatorname{diag}\left[\mathbf{G}\left(l_{1}: l_{2}\right) \cdots \mathbf{G}\left(l_{1}: l_{2}\right)\right]$.

If the above condition holds for some users but not for others, one may need to re-select $\mathbf{X}\left(l_{1}: l_{2}\right)$ and/or increase the matrix's dimension. However, our extensive simulations show that as long as the necessary conditions in Theorem 3 are satisfied, channels are generally identifiable for all the users.

Two naturally arising questions concern i) the length of the reduced dimension observation vectors and ii) if there exists an optimal choice for a given dimension. While the length of the observation vectors is a trade-off between performance and computational burden, a lower bound is given by Theorem 3 as $d_{r} \geq d_{c}+M_{i} /\left(m q_{i}+1\right)$. For a given observation vector dimension, determining the location that yields best performance necessitates performance analysis, the key result for which is summarized next.

Result: Denote the estimation error $\Delta \mathbf{h}_{i j}=\hat{\mathbf{h}}_{i j}-\mathbf{h}_{i j}$; the mean square error (MSE) is then approximated by

$$
\operatorname{MSE}=E\left(\Delta \mathbf{h}_{i j}^{H} \Delta \mathbf{h}_{i j}\right) \simeq \frac{\sigma_{w}^{2}}{J \sigma_{i j}^{2}}\left\|\mathcal{Q}_{i j}^{\dagger}\right\|_{F}^{2} .
$$

Proof: See the Appendix. 
It is easy to verify that the sample covariance matrix $\hat{\mathbf{R}}=$ $\mathbf{X X}^{H} / J$ is an unbiased estimator of the true covariance matrix $\mathbf{R}=E\left(\mathbf{x}_{n} \mathbf{x}_{n}^{H}\right)$. From central limit theorem we know that as $J \rightarrow \infty$, the distribution of the perturbation to $\mathbf{R}, \Delta \mathbf{R}=$ $\hat{\mathbf{R}}-\mathbf{R}$, approaches Gaussian distribution of zero mean and variance of order $O(1 / J)$, i.e., the result above provides a first-order approximation to the true MSE that is accurate for sufficiently large $J$.

Note from (37) that MSE depends on both spreading codes and channels; thus it is not possible in general to determine the optimal value of $l_{1}$ in advance without knowledge of the channel. Our simulation in Section V however shows that the choice of $l_{1}$ is not critical, at least for the channels considered.

\section{Simulation Results}

In order to assess performance of the proposed methods for multi-rate CDMA systems, a commensurate single rate system must be first determined. Thus we established the following baseline toward that end-

1) System Bandwidth: The single rate, multi-code and variable processing gain systems have the same chip duration $T_{c}$ (and hence same bandwidth) as the highest rate users in variable chip rate systems.

2) Net Rate Budget: The total data rate (bits/s) is conserved in all systems, i.e.,

$$
\sum_{i=1}^{N} \frac{K_{i}}{T_{i}}=\frac{K}{T_{s r}}
$$

where $K=\sum_{i=1}^{N} K_{i}$ is the total number of users in any system, and $T_{s r}$ is the symbol duration of the equivalent single rate system.

3) Identical duration of observation in all systems.

4) Identical length of observation vector $\mathbf{x}_{n}$ in all cases, achieved by suitable choice of the smoothing factor.

5) Same two-ray multipath physical channel

$$
f_{i j}(t)=\delta(t)-0.7 \delta\left(t-T_{c} / 3\right)
$$

for all users in the system; the composite channel $h_{i j}(t)$ is the convolution of $f_{i j}(t)$ and raised-cosine pulse shaping function with roll-off factor 0.10 (with respect to the reciprocal of the user's chip duration); $h_{i j}(t)$ is truncated to duration $\left[-3 T_{c}, 3 T_{c}\right]$ in the single rate, multi-code and variable processing gain systems and to $\left[-3 T_{c}^{i}, 3 T_{c}^{i}\right]$ in the variable chip rate system. Thus the channel orders are 5 for the single rate, multi-code and variable processing gain systems, 11 for high rate users and 17 for low rate users in the variable chip rate system.

6) Randomly generated binary spreading codes used in all the systems (including multi-code) to eliminate any dependence of performance on code correlations.

Estimator performance is evaluated in terms of normalized root mean square error (NRMSE), defined as

$$
\text { NRMSE }=\sqrt{\frac{1}{D} \sum_{d=1}^{D}\left\|\hat{\mathbf{h}}_{d}-\frac{\mathbf{h}}{\|\mathbf{h}\|}\right\|^{2}}
$$

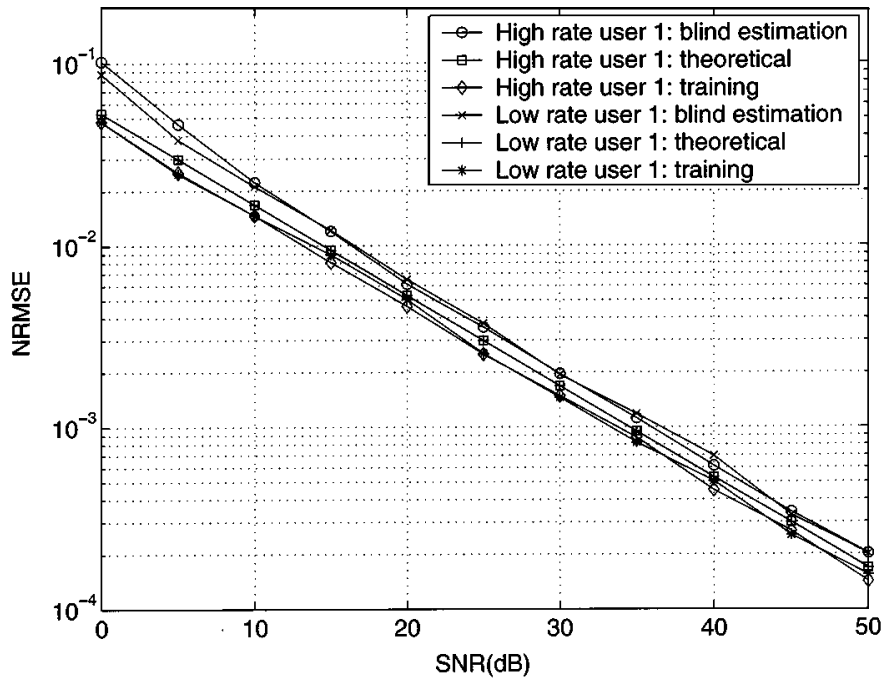

Fig. 4. NRMSE versus SNR with full dimension method for the variable processing gain system.

where $D$ is the total number of Monte Carlo runs, $\hat{\mathbf{h}}_{d}$ is the channel estimate at the $d$ th run, $\mathbf{h}$ is the true channel and it is normalized to remove the scalar ambiguity of the estimates.

The desired user's (average) signal-to-noise (SNR) ratio is defined as

$$
\mathrm{SNR}=\frac{\left\|\mathrm{g}_{i j}\right\|^{2} \sigma_{i j}^{2} / L_{i}}{\sigma_{w}^{2}} .
$$

Example 1 (Full Dimension Method): We consider a dualrate system of 6 active users, 3 at high rate and 3 at low rate. The rate ratio is $2: 3$. In the variable processing gain system, we choose $L=5$, thus the processing gains for high rate and low rate users are 10 and 15 respectively. Let $m=2$, implying that the observation vector length is 60 . Data is obtained for transmission duration of $300 p_{1} T_{2}=300 p_{2} T_{1}$ (therefore 900 symbols transmitted at high rate and 600 symbols at low rate) in each independent trial. Using the equivalence conditions above, the following corresponding settings for the multi-code system are obtained: processing gain 30 , smoothing factor 2,300 symbols transmitted by each of the 15 virtual users. For the variable chip rate system the configurations are: processing gain 10 [thus effective processing gains are 20 and 30 respectively, see (21)], smoothing factor 1, 900 symbols transmitted at high rate and 600 symbols transmitted at low rate. For the single rate system the specifications are: processing gain 12; smoothing factor 5, 750 symbols transmitted by each of the 6 users. Note that the above parameter settings satisfy the necessary conditions described in Theorem 1. We assume channel orders are known a priori and conduct 100 independent runs to compute the NRMSE under perfect power control, i.e., all users have equal transmit power. The simulation results are presented in Figs. 4-6, along with the theoretical results computed according to (37) and training-based LS estimation curves. We can see that in all three multi-rate systems, 1) the theoretical expression well approximates the simulation results; 2) minor estimation degradation is induced when the blind method is used instead of the training-based approach. It is also observed that high rate and low rate users have comparable NRMSE in the multi-code 


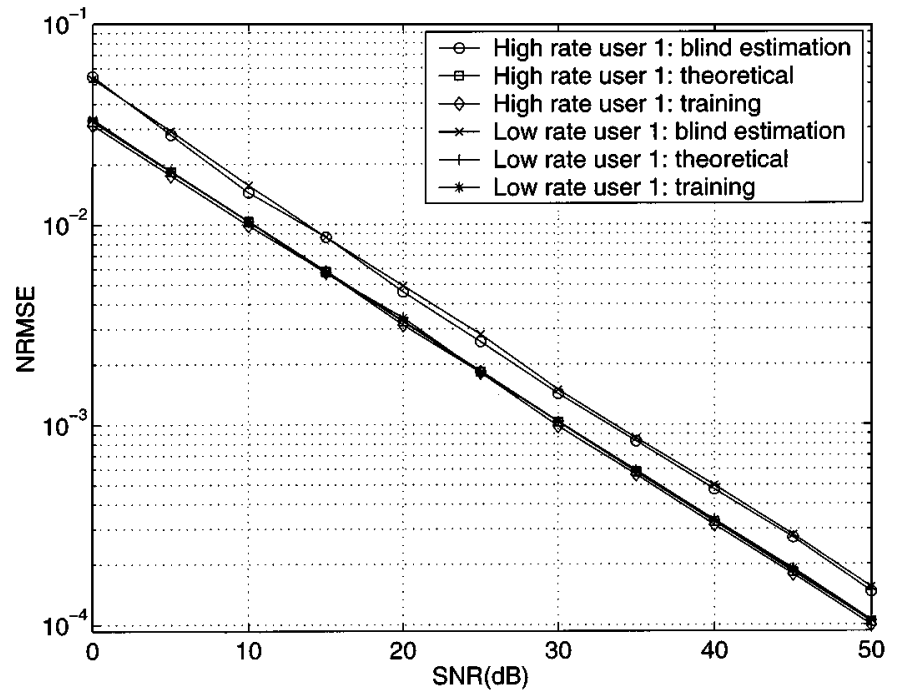

Fig. 5. NRMSE versus SNR with full dimension method for the multi-code system.

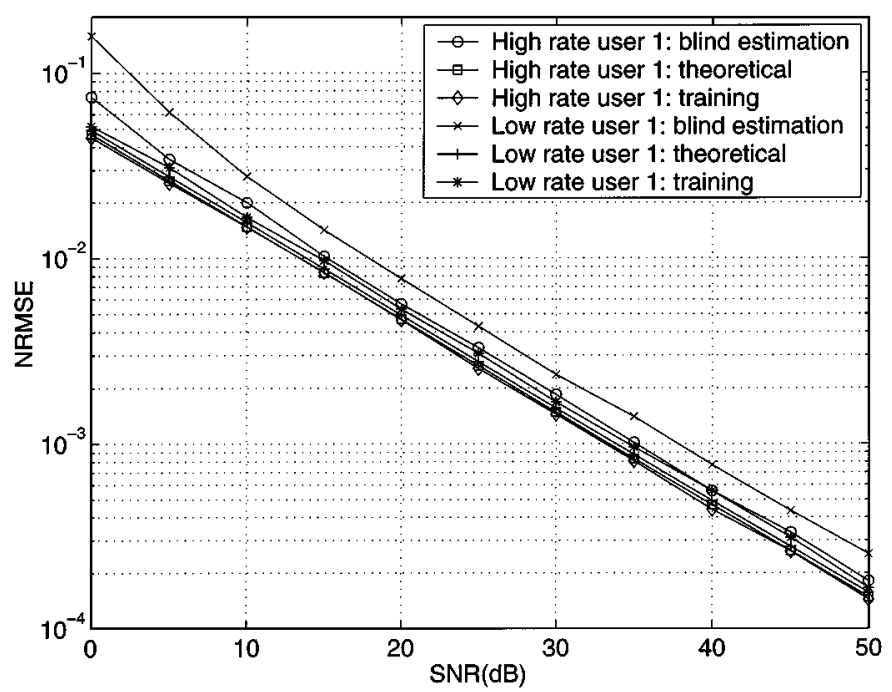

Fig. 6. NRMSE versus SNR with full dimension method for the variable chip rate system.

and variable processing gain systems, in contrast to the variable chip rate system where high rate users have better estimation performance. This is due to the fact that low rate users have a larger channel order in the variable chip rate system. The single rate system performance vis-a-vis the three multi-rate systems is shown in Fig. 7 where average NRMSE's over rates are used for multi-rate systems. It is observed that for the simulation settings, all the systems are comparable while the multi-code system is slightly superior to others.

Example 2 (Reduced Complexity Method): The performance/complexity tradeoffs for the reduced complexity method are investigated. In contrast to example 1 , there are 5 high rate and 5 low rate users in the variable processing gain system, $L=10$ and $100 p_{1} T_{2}=100 p_{2} T_{1}$ observation duration is used. The rate ratio is again $2: 3$ and $m=2$. The parameters for other systems are computed accordingly. Simulation results are presented in Figs. 8 and 9. This method is parametrized by a "location" variable $l_{1}$ and the (reduced) vector dimension $d_{r}$. The minimum dimension of the observation vector is based on

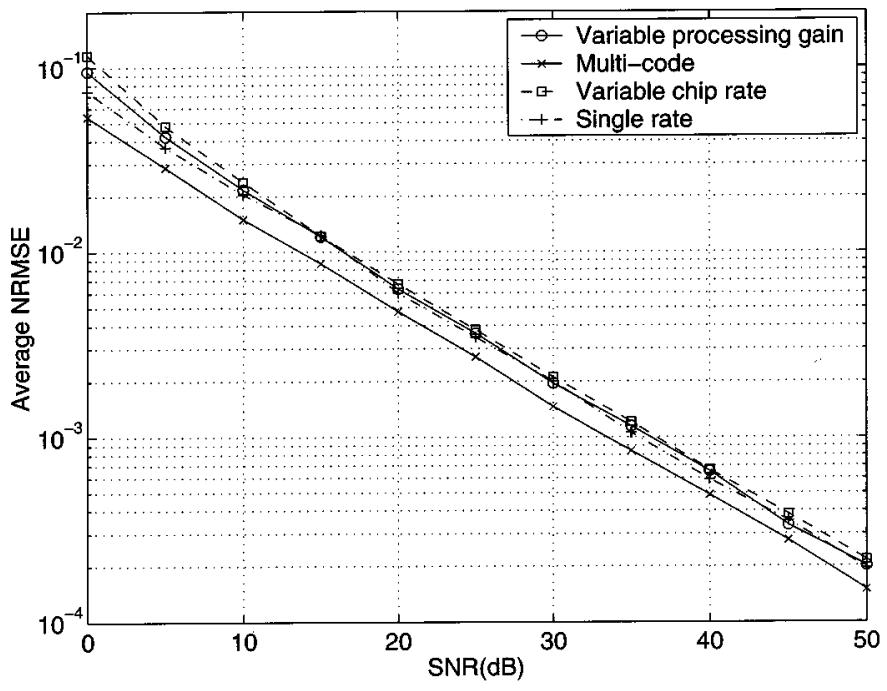

Fig. 7. Estimation performance comparison.

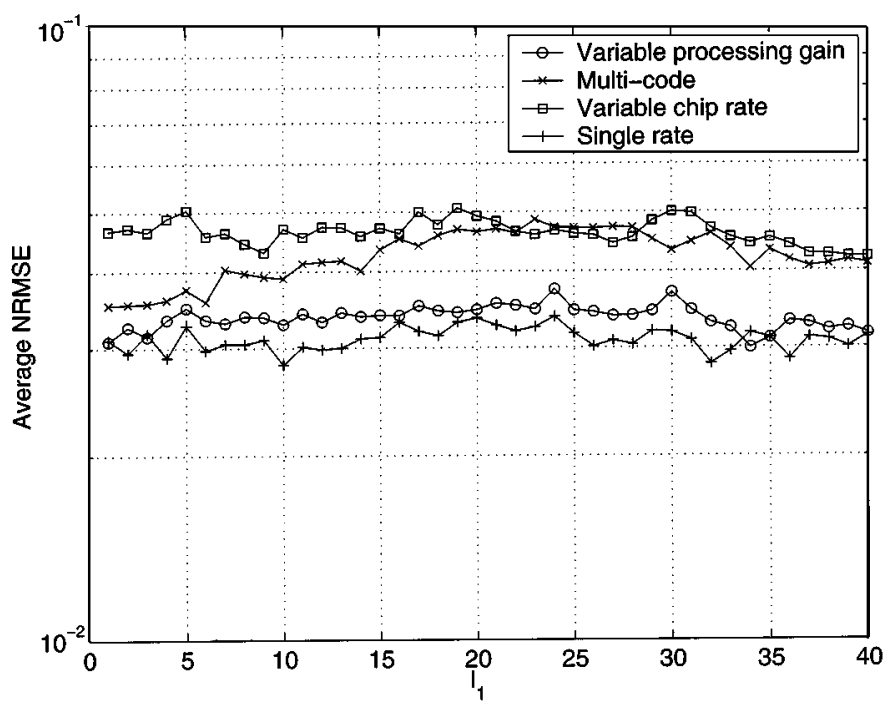

Fig. 8. Average NRMSE versus $l_{1}$.

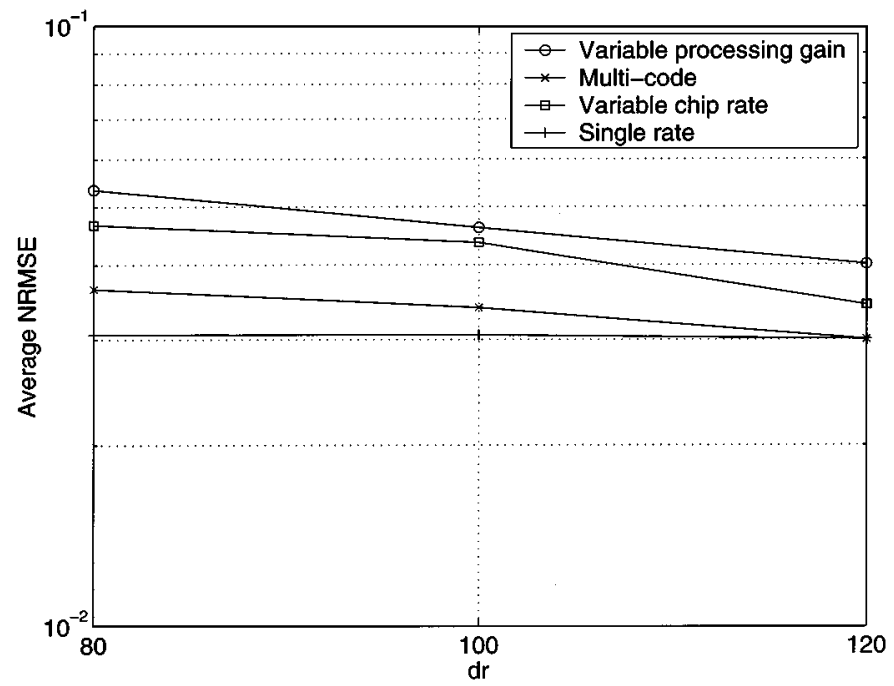

Fig. 9. Average NRMSE versus vector length.

Theorem 3; that requires knowledge of $d_{c}$. To obviate a search for this parameter, we replace $d_{c}$ by the number of columns in 


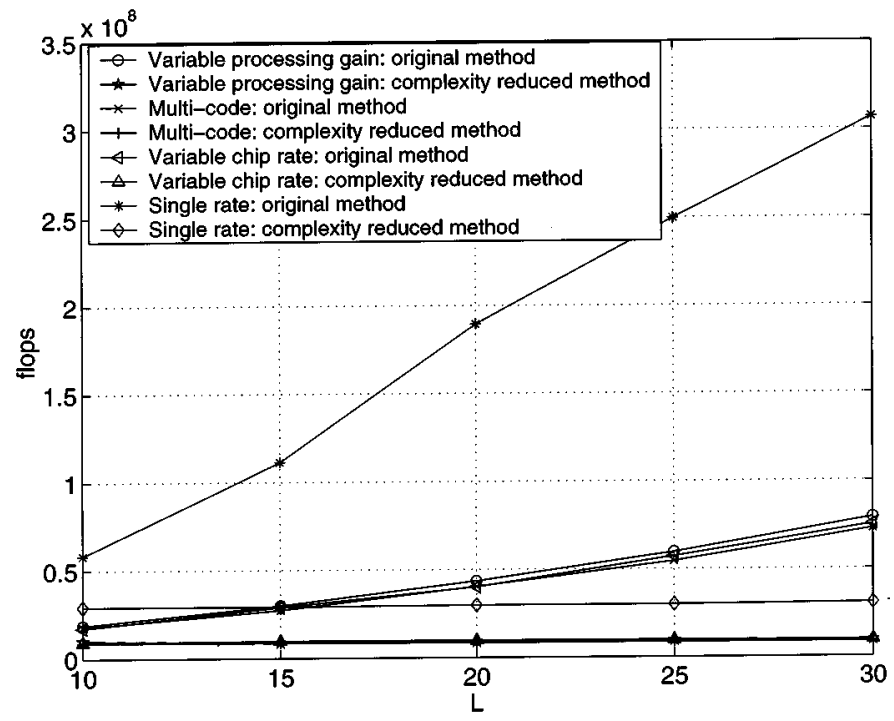

Fig. 10. Complexity versus $L$.

G that constitutes a ready upper bound. Thus the observation vector dimensions are no less than 61 in variable processing gain, 77 in multi-code, 41 in variable chip rate systems and 61 in the single rate system respectively. We choose $d_{r}=80$ and test the performance of the reduced complexity method with every possible value of $l_{1}$. Perfect power control is assumed. Fig. 8 is the plot of the average NRMSE over rates as a function of $l_{1}$ with SNR $=5 \mathrm{~dB}$ and known channel orders. The result shows that performance sensitivity to $l_{1}$ is not significant, thus in the remainder of this example, $l_{1}=1$ is used. With other simulation parameters fixed as before, Fig. 9 illustrates the effect of vector length on the average NRMSE, which is seen to be monotonically decreasing function of $d_{r}$, highlighting the trade-off between estimation accuracy and complexity. Estimates of the relative computational complexity are shown in Fig. 10 in terms of flop counts for the SVD for the respective methods versus $L$ as defined by (5); the processing gains of other systems are computed according to conditions 1), 2) of the equivalence baseline. Observation vector length $d_{r}=80$ is used in the reduced complexity method to estimate the computational demands. Note that this value satisfies the necessary conditions of Theorem 3 independent of $L$. It is seen that as the processing gains increase, the complexity of the modified method is invariant to $L$ (the three multi-rate systems have nearly identical complexity) in contrast to the original method-this is because the row dimension of $\mathbf{X}$ increases while that of $\mathbf{X}\left(l_{1}: l_{2}\right)$ does not. Further, the complexity of the single rate system for the original method is much larger than those of multi-rate systems for large $L$. While the complexity of both the single rate and multi-rate systems is $O\left(L^{2}\right)$ as anticipated, the (multiplicative) constant differs due to the fact that the number of columns in $\mathbf{X}$ for the single rate system is larger than the corresponding multi-rate cases. We conclude that significant computation savings can be achieved with our modified method, particularly for large $L$.

Example 3 (Channel Order Selection): In this example we evaluate the effect of channel order overestimation. Simulation scenario is exactly the same as that in Example 2, except that

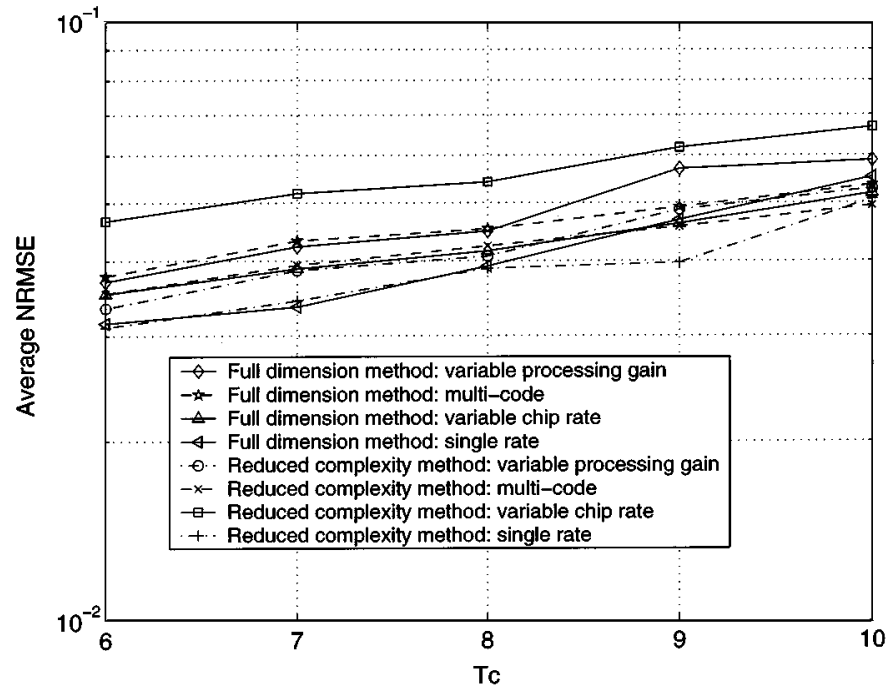

Fig. 11. The effect of channel order overestimation on average NRMSE.

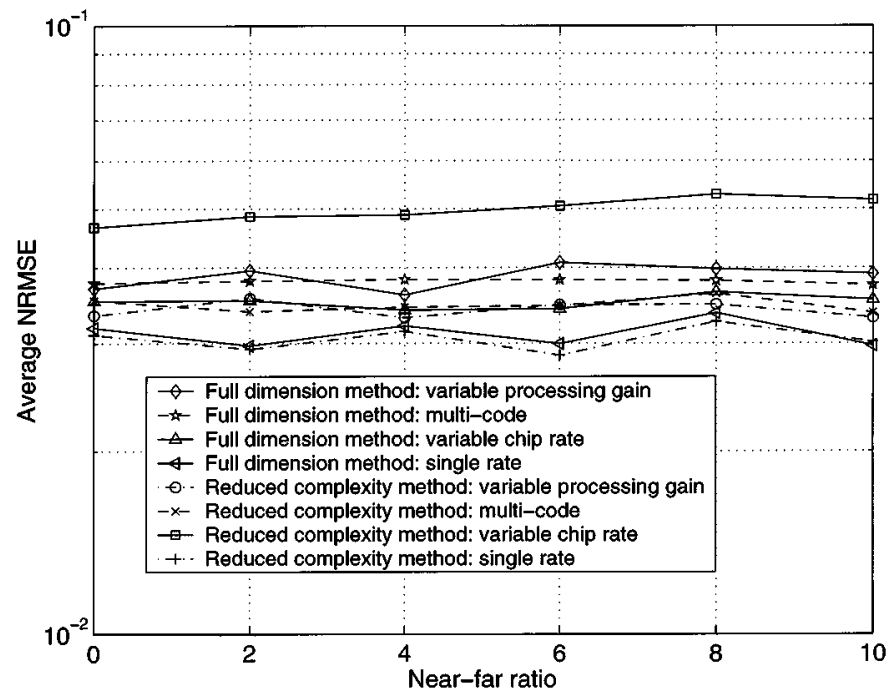

Fig. 12. Near-far effect.

SNR is fixed at $5 \mathrm{~dB}$ and channel order estimate measured in chip duration is varying. In addition, $l_{1}=1, d_{r}=80$ for the reduced complexity method. Note that the true order is 6 chips and the corresponding true $M_{i}$ is specified by the equivalence condition 5) at the beginning of this section. The average NRMSE curves in Fig. 11 show that overestimation of channel orders results in minor NRMSE increase, attesting to its robustness.

Example 4 (Near-Far Effect): CDMA systems are interference limited since the received signature sequences of different users are not orthogonal; thus strong (undesired) users may overshadow weak (desired) users, resulting in the near-far problem. In such cases, multi-user (or interference suppressive) detection is employed to eliminate the multi-user interference whose performance depends critically on the accuracy of the signature waveform estimation. Once again, we use parameters from Example 2 with desired user's SNR $=5 \mathrm{~dB}$; as before, $l_{1}=1, d_{r}=80$ for the reduced complexity method. In multi-rate systems we assume two desired users, one at high 


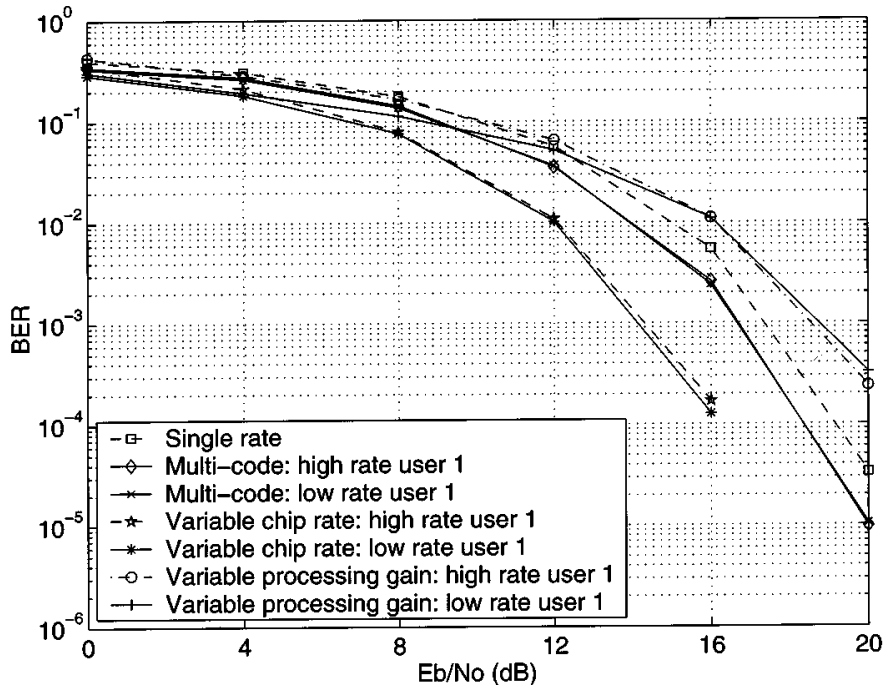

Fig. 13. BER performance.

rate and one at low rate; in the single rate system we only assume one desired user. We also assume that all interfering users have the same signal power. Clearly, the average NRMSE in Fig. 12 is largely invariant to near-far ratio (ratio of the interfering users' signal power to the desired user's) as anticipated, since the signal subspace is determined by the users' normalized signature waveforms and not their powers.

Example 5 (BER Performance): Based on the data model (15), we construct the decorrelating receivers for the systems specified in Example 1 with channel estimates obtained via full dimension method. For uncoded BPSK modulation, the BER curves are shown in Fig. 13, where we have assumed user 1 at each rate is the desired user. It can be seen that the variable chip rate system has the best performance and the multi-code system outperforms the single rate system while the latter is superior to the variable processing gain system. The exception is that the low rate user in the variable processing gain system has better performance than those of the multi-code and single rate system in low SNR region.

\section{CONCLUSION}

A unified model has been introduced for three multi-rate CDMA access methods over dispersive channels. A subspace based channel estimation algorithm developed for possible use in coherent multi-rate detectors requires complexity essentially determined by $L P$, the least common multiple of the system's processing gains, which is typically much larger than any individual user's processing gain. A modified method using reduced dimension observation vectors is then introduced that results in considerable complexity reduction (vs. performance trade-offs) as illustrated by simulations. Investigation shows that estimation performance does not change much as the location of the reduced observation vectors varies; thus only the dimension of the reduced observation vectors needs to be determined, which can be adapted in response to performance requirements. We remark that the important problem of effective multi-rate multi-user detector design needs significant additional work that must be deferred to the future.

\section{APPENDIX \\ MSE OF THE CHANNEL ESTIMATOR}

The derivation is based on first order perturbation theory introduced in [16]. We use the same method as [17] to obtain the explicit form for MSE. $\mathcal{U}_{w}$ denotes the true complementary subspace of $\mathbf{G}\left(l_{1}: l_{2}\right)$ and introduce $\Delta \mathcal{U}_{w}\left(\Delta \mathcal{Q}_{i j}\right)$ as the difference between $\mathcal{U}_{w}\left(\mathcal{Q}_{i j}\right)$ and its estimate. From [16], the perturbation to $\mathcal{U}_{w}$ due to noise is given by

$$
\Delta \mathcal{U}_{w} \simeq-\mathbf{X}^{\dagger}\left(l_{1}: l_{2}\right) \mathbf{W}^{H}\left(l_{1}: l_{2}\right) \mathcal{U}_{w} .
$$

Introducing the following block diagonal matrices

$$
\begin{aligned}
& \mathcal{X}=\underbrace{\left[\begin{array}{lll}
\mathbf{X}^{\dagger}\left(l_{1}: l_{2}\right) & & \\
& \ddots & \\
& & \mathbf{X}^{\dagger}\left(l_{1}: l_{2}\right)
\end{array}\right]}_{m q_{i}+1 \text { blocks }} \\
& \mathcal{W}=\underbrace{\left[\begin{array}{lll}
\mathbf{W}\left(l_{1}: l_{2}\right) & & \\
& \ddots & \\
& & \mathbf{W}\left(l_{1}: l_{2}\right)
\end{array}\right]}_{m q_{i}+1 \text { blocks }}
\end{aligned}
$$

we obtain

$$
\Delta \mathcal{Q}_{i j}=\tilde{\mathcal{C}}_{i j}^{H} \Delta \tilde{\mathcal{U}}_{w} \simeq-\tilde{\mathcal{C}}_{i j}^{H} \mathcal{X} \mathcal{W}^{H} \tilde{\mathcal{U}}_{w}
$$

Similarly, the perturbation to the channel estimation assumes the form

$$
\Delta \mathbf{h}_{i j} \simeq-\mathcal{Q}_{i j}^{\dagger} \Delta \mathcal{Q}_{i j}^{H} \mathbf{h}_{i j} \simeq \mathcal{Q}_{i j}^{\dagger} \tilde{\mathcal{U}}_{w}^{H} \mathcal{W} \mathcal{X}^{H} \tilde{\mathcal{C}}_{i j} \mathbf{h}_{i j}
$$

Let $\mathbf{e}_{k}$ be the column vector with $k$ th element one and zero otherwise, the $k$ th element of $\Delta \mathbf{h}_{i j}$ is given by $\Delta \mathbf{h}_{i j}(k)=\mathbf{e}_{k}^{H} \mathbf{h}_{i j}$. Thus

$$
\begin{aligned}
E\left\{\Delta \mathbf{h}_{i j}^{H}(k) \Delta \mathbf{h}_{i j}(k)\right\} & \simeq E\left\{\left\|\mathbf{e}_{k}^{H} \mathcal{Q}_{i j}^{\dagger} \tilde{\mathcal{U}}_{w}^{H} \mathcal{W} \mathcal{X}^{H} \tilde{\mathcal{C}}_{i j} \mathbf{h}_{i j}\right\|^{2}\right\} \\
& =\sigma_{w}^{2} \mathbf{e}_{k}^{H} \mathcal{Q}_{i j}^{\dagger} \mathbf{Z} \mathcal{Q}_{i j}^{\dagger} \mathbf{e}_{k} .
\end{aligned}
$$

Denote $N_{w}$ the number of column vectors in $\mathcal{U}_{w}$, the $\left(m q_{i}+1\right) N_{w} \times\left(m q_{i}+1\right) N_{w}$ block matrix $\mathbf{Z}$ is constructed with $\left(b_{r}, b_{c}\right)$ th $\left(\left(1<b_{r}, b_{c}<m q_{i}+1\right)\right)$ block defined as $\mathbf{h}_{i j}^{H}\left(\mathcal{C}_{i j}^{b_{c}}\left(l_{1}: l_{2}\right)\right)^{H} \mathbf{X}^{\dagger}\left(l_{1}: l_{2}\right) \mathbf{X}^{\dagger^{H}}\left(l_{1}: l_{2}\right) \mathcal{C}_{i j}^{b_{r}}\left(l_{1}: l_{2}\right) \mathbf{h}_{i j} \mathbf{I}_{N_{w}}$. From [16, (73) and (80)] we know that

$$
\begin{aligned}
\mathbf{G}^{H}\left(l_{1}: l_{2}\right) \mathbf{X}^{\dagger}\left(l_{1}: l_{2}\right) \mathbf{X}^{\dagger^{H}}\left(l_{1}: l_{2}\right) \mathbf{G}\left(l_{1}: l_{2}\right) \\
=\left(\mathbf{S S}^{H}\right)^{-1} \simeq \frac{\left(E\left\{\mathbf{s}_{n} \mathbf{s}_{n}^{H}\right\}\right)^{-1}}{J}=\frac{\mathbf{R}_{s}^{-1}}{J} .
\end{aligned}
$$

Since $\mathcal{C}_{i j}^{b_{r}}\left(l_{1}: l_{2}\right) \mathbf{h}_{i j}$ and $\mathcal{C}_{i j}^{b_{c}}\left(l_{1}: l_{2}\right) \mathbf{h}_{i j}$ are two column vectors of $\mathbf{G}_{i j}\left(l_{1}: l_{2}\right)$ and hence of $\mathbf{G}\left(l_{1}: l_{2}\right)$, suppose they are indexed as the $r_{1}$ th and $r_{2}$ th then

$$
\begin{aligned}
\mathbf{h}_{i j}^{H}\left(\mathcal{C}_{i j}^{b_{c}}\left(l_{1}: l_{2}\right)\right)^{H} \mathbf{X}^{\dagger}\left(l_{1}: l_{2}\right) \mathbf{X}^{\dagger^{H}} \mathcal{C}_{i j}^{b_{r}} & \left(l_{1}: l_{2}\right) \mathbf{h}_{i j} \\
& \simeq \frac{\mathbf{R}_{s}^{-1}\left(r_{1}, r_{2}\right)}{J}
\end{aligned}
$$


where $\mathbf{R}_{s}^{-1}\left(r_{1}, r_{2}\right)$ is the $\left(r_{1}, r_{2}\right)$ th element of $\mathbf{R}_{s}^{-1}$, which is $1 / \sigma_{i j}^{2}$ if $r_{1}=r_{2}$ and zero otherwise. So $\mathbf{Z}=\mathbf{I}_{\left(m q_{i}+1\right) N_{w}} / J \sigma_{i j}^{2}$ which indicates

$$
E\left\{\Delta \mathbf{h}_{i j}^{H}(k) \Delta \mathbf{h}_{i j}(k)\right\} \simeq \frac{\sigma_{w}^{2}\left\|\mathbf{e}_{k}^{H} \mathcal{Q}_{i j}^{\dagger}\right\|^{2}}{J \sigma_{i j}^{2}}
$$

and

$$
\begin{aligned}
E\left\{\Delta \mathbf{h}_{i j}^{H} \Delta \mathbf{h}_{i j}\right\} & \simeq \sum_{k} \frac{\sigma_{w}^{2}\left\|\mathbf{e}_{k}^{H} \mathcal{Q}_{i j}^{\dagger}\right\|^{2}}{J \sigma_{i j}^{2}} \\
& =\frac{\sigma_{w}^{2}}{J \sigma_{i j}^{2}}\left\|\mathcal{Q}_{i j}^{\dagger}\right\|_{F}^{2} .
\end{aligned}
$$

\section{ACKNOWLEDGMENT}

H. Yan would like to thank Dr. K. Li for helpful discussions on the work presented herein.

\section{REFERENCES}

[1] T. Ojanpera and R. Prasad, "Overview of air interface multiple access for IMT-2000/UMTS,” IEEE Commun. Mag., vol. 36, pp. 82-86, Sept. 1998.

[2] M. Zeng, A. Annamalai, and V. K. Bhargava, "Harmonization of global third-generation mobile systems," IEEE Commun. Mag., vol. 38, pp. 94-104, Dec. 2000.

[3] C.-L. I and R. D. Gitlin, "Multi-code CDMA wireless personal communications networks," in Proc. ICC, vol. 2, June 1995, pp. 1060-1064.

[4] T.-H. Wu and E. Geraniotis, "CDMA with multiple chip rates for multimedia communications," in Proc. 1994 Conf. Inf. Sci. \& Syst., Baltimore, MD, 1994, pp. 992-997.

[5] T. Ottosson and A. Svensson, "Multi-rate schemes in DS/CDMA systems," in Proc. IEEE Vehic. Tech. Conf., Chicago, July 1995, pp. 1006-1010.

[6] S. Ramakrishna and J. Holtzman, "A comparison between single code and multiple code transmission schemes in a CDMA system," in Proc. IEEE Vehic. Tech. Conf., Ottawa, ON, Canada, May 1998, pp. 791-795.

[7] M. Saquib, R. Yates, and N. Mandayam, "Decorrelating detectors for a dual rate synchronous DS/CDMA system," Wireless Personal Commun., vol. 9, no. 3, pp. 197-216, May 1999.

[8] —, "A decision feedback decorrelator for a dual rate synchronous DS/CDMA system," Wireless Networks, vol. 4, pp. 497-506, 1998.

[9] M. Saquib, R. Yates, and A. Ganti, "An asynchronous multirate decorrelator," IEEE Trans. Commun., vol. 48, pp. 739-742, May 2000.

[10] A.-L. Johansson and A. Svensson, "Successive interference cancellation in multiple data rate DS/CDMA systems," in Proc. IEEE Vehic. Tech. Conf., Chicago, IL, July 1995, pp. 704-708.

[11] C. Wijting, T. Ojanpera, M. Juntti, K. Kansanen, and R. Prasad, "Groupwise serial multiuser detectors for multirate DS-CDMA," in Proc. IEEE Vehic. Tech. Conf., Amsterdam, The Netherlands, Sept. 1999, pp. 836-840.

[12] A.-L. Johansson, "Group-wise successive interference cancellation in multirate CDMA systems," in Proc. IEEE Vehic. Tech. Conf., Amsterdam, The Netherland, Sept. 1999, pp. 1435-1439.

[13] U. Mitra, "Observations on jointly optimal detection for multi-rate DS/CDMA systems," in Proc. IEEE GLOBECOM Commun. Theory Mini Conf., London, U.K., Nov. 1996, pp. 116-120.

[14] J. Chen and U. Mitra, "Optimum near-far resistance for dual-rate DS/CDMA signals: Random signature sequence analysis," IEEE Trans. Inform. Theory, vol. 45, pp. 2434-2447, Nov. 1999.
[15] U. Mitra, "Comparison of maximum likelihood based detection for two multirate access schemes for CDMA signals," IEEE Trans. Commun., vol. 47, pp. 64-77, Jan. 1999.

[16] F. Li, H. Liu, and R. J. Vaccaro, "Performance analysis for DOA estimation algorithms: Unification, simplification, and observations," IEEE Trans. Aeorsp. Electron. Syst., vol. AES-29, pp. 1170-1184, Oct. 1993.

[17] H. Liu and G. Xu, "A subspace method for signature waveform estimation in synchronous CDMA systems," IEEE Trans. Commun., vol. 44, pp. 1346-1354, Oct. 1996

[18] M. Torlak and G. Xu, "Blind multiuser channel estimation in asynchronous CDMA systems," IEEE Trans. Signal Processing, vol. 45, pp. 137-147, Jan. 1997.

[19] J. G. Proakis, Digital Communications, 3rd ed. New York: McGrawHill, 1995.

[20] T. S. Rappaport, Wireless Communications: Principles \& Practice. Englewood Cliffs, NJ: Prentice-Hall, 1996.

[21] I. Satake, Linear Algebra. New York: Marcel Dekker, 1975.

[22] L. Tong, G. Xu, and T. Kailath, "Blind identification and equalization based on second order statistics: A time domain approach," IEEE Trans. Inform. Theory, vol. 40, pp. 340-349, Mar. 1994.

[23] E. Moulines, P. Duhamel, J. Cardoso, and S. Mayrargue, "Subspace methods for the blind identification of multichannel FIR filters," IEEE Trans. Signal Processing, vol. 43, pp. 516-525, Feb. 1995.

[24] S. Talwar, M. Viberg, and A. Paulraj, "Blind estimation of multiple co-channel digital signals using an antenna array," IEEE Signal Processing Lett., vol. 1, pp. 29-31, Feb. 1994.

[25] G. Golub and C. Van Loan, Matrix Computations, 3rd ed. Baltimore, MD: Johns Hopkins Univ. Press, 1995.

[26] G. Strang, Linear Algebra and Its Applications, 3rd ed. San Diego, CA: Harcourt Brace Jovanovich, 1988.

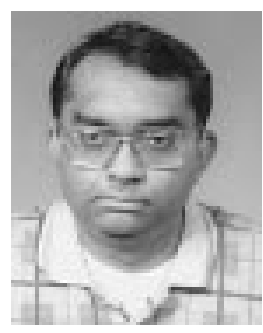

Sumit Roy (S'84-M'88-SM'00) received the B.Tech. degree from the Indian Institute of Technology (Kanpur) in 1983 and the M.S. and Ph.D. degrees from the University of California (Santa Barbara) in 1985 and 1988, respectively, all in electrical engineering, and the M.A. degree in statistics and applied probability in 1988.

His previous academic appointments were at the Moore School of Electrical Engineering, University of Pennsylvania, and at the University of Texas, San Antonio. He is presently Associate Professor of Electrical Engineering, University of Washington, Seattle, where his research interests include analysis/design of communication systems/networks, with a topical emphasis on next generation mobile/wireless networks. He is currently on academic leave at Intel Wireless Technology Lab working on ultrawide-band radios for high-speed wireless access.

Dr. Roy's activities for the IEEE Communications Society includes membership of several technical committees and conference program committees, and he presently serves as an Editor for the new publication, IEEE TRANSACTIONS ON WIRELESS COMMUNICATIONS.

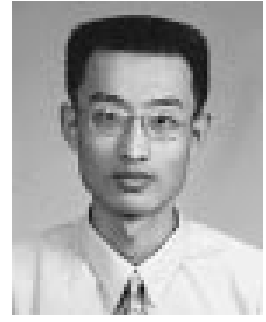

Hongbo Yan received the B.S. degree from Northwestern Polytechnic University, Xi'an, China, in 1995 and the M.S. degree from Tsinghua University, Beijing, China, in 1998. He is currently working toward the Ph.D. degree in the Department of Electrical Engineering, the University of Washington, Seattle.

During the summer of 1998, he was with AT\&T Wireless Services, Inc., where he worked on fixed wireless systems. His current research interests are wireless communications with emphasis on CDMA 